\title{
Correction to: Local directional gradient pattern: a local descriptor for face recognition
}

\author{
Soumendu Chakraborty ${ }^{1} \cdot$ Satish Kumar Singh $^{1}$ • \\ Pavan Chakraborty ${ }^{1}$
}

Published online: 18 January 2018

C) Springer Science+Business Media, LLC, part of Springer Nature 2018

\section{Correction to: Multimed Tools Appl (2017) 76:1201-1216 \\ https://doi.org/10.1007/s11042-015-3111-6}

The Eqs. 3 and 15 in the original version of this article contained an error. The correct equations are presented below:

$$
\begin{gathered}
I_{0^{\circ}}^{\prime}\left(\mathbb{R}_{0}\right)=I\left(\mathbb{R}_{0}\right)-I\left(\mathbb{R}_{1}\right) \\
C\left(I_{\theta}^{n-1}\left(\mathbb{R}_{0}\right), I_{\alpha}^{n-1}\left(\mathbb{R}_{0}\right)\right)= \begin{cases}1, & \text { if } I_{\theta}^{n-1}\left(\mathbb{R}_{0}\right)>I_{\alpha}^{n-1}\left(\mathbb{R}_{0}\right) \\
0, & \text { else }\end{cases}
\end{gathered}
$$

The online version of the original article can be found at https://oi.org/10.1007/s11042-015-3111-6.

Soumendu Chakraborty

soum.uit@gmail.com

Satish Kumar Singh

sk.singh@iiita.ac.in

Pavan Chakraborty

Pavan@iiita.ac.in

1 Department of Information Technology, Indian Institute of Information Technology, Allahabad, India 